Palabra Clave (La Plata), abril 2017, vol. 6, n² 2, e023. ISSN 1853-9912

Universidad Nacional de La Plata.

Facultad de Humanidades y Ciencias de la Educación.

Departamento de Bibliotecología

\title{
Redesastres, una herramienta de gestión de información al servicio de la reducción de desastres sanitarios en animales y plantas en Cuba
}

\author{
Disaster management tool serving information \\ reducing health disasters in animals and plants in \\ Cuban
}

\section{Haymeé Canales $*$, María Irian Percedo $* *$, Mayra G. Rodríguez $* * *$, Isel González $* * * *$}

* Grupo Gestión de Información, Centro Nacional de Sanidad Agropecuaria (CENSA), ** Dirección de Salud Animal, Centro Nacional de Sanidad Agropecuaria (CENSA), *** Dirección de Protección Vegetal, Centro Nacional de Sanidad Agropecuaria (CENSA), **** Grupo Informática y Redes, Centro Nacional de Sanidad Agropecuaria (CENSA), Cuba I haymee@censa.edu.cu,percedo@censa.edu.cu,mrguez@censa.edu.cu, isel@censa.edu.cu

\section{PALABRAS CLAVE RESUMEN}

Gestión de Información

El presente artículo tiene por objetivo divulgar las experiencias del trabajo de gestión de información en REDesastres, primera red telemática del Centro de Capacitación para la Reducción de Desastres Sanitarios en Animales y Plantas (CEDESAP), adscrito al Centro Nacional de Sanidad Agropecuaria (CENSA) y auspiciado por el Ministerio de Educación Superior (MES) y el Estado Mayor Nacional de la Defensa Civil (EMNDC) de Cuba. REDesastres brinda soporte informacional a todos los miembros de la red como factor clave para la actualización, la toma de decisiones oportunas y la participación activa de todos los actores y sectores involucrados en la reducción de desastres sanitarios en animales y plantas. Cuenta con más de 510 destinos y posibilita la interconexión en tiempo real de profesionales, directivos y funcionarios de diversas disciplinas e instituciones cubanas y latinoamericanas. A través de la red han circulado más de 1.376 mensajes con informaciones relevantes, actualizadas y comentadas, provenientes de organismos sanitarios y agencias noticiosas internacionales, publicaciones científicas y fuentes nacionales, relativas a las enfermedades emergentes, reemergentes, transfronterizas y la gestión de desastres.

\section{KEYWORDS ABSTRACT}

Information management

The present article aims to disseminate the experiences of the information management work in REDesastres, the first telematic network of the Training Center for the Reduction of Animal and Plant Health Disasters (CEDESAP), attached to the National Center for Agricultural and Livestock Health (CENSA) and Sponsored by the Ministry of Higher Education (MES) and the National Civil Defense National Staff (EMNDC) of Cuba. REDesastres provides informational support to all members of the network as a key factor for updating, timely decision making and active participation of all actors and sectors involved in the reduction of animal and plant health disasters. It has more than 510 destinations and enables the real-time interconnection of professionals, executives and officials from various Cuban and Latin American disciplines and institutions. Through the network, more than 1.376 messages have been circulated with relevant up-to-date and commented information from health agencies and international news agencies, scientific publications and national sources on emerging, reemerging, transboundary diseases and disaster management.

Recibido: 2 de diciembre de 2016 | Aceptado: 27 de marzo de 2017 | Publicado: 20 de abril de 2017

Cita sugerida: Canales, H., Percedo, M., Rodríguez, M. y González, I. (2017). Redesastres, una herramienta de gestión de información al servicio de la reducción de desastres sanitarios en animales y plantas en Cuba. Palabra Clave (La Plata), 6(2), e023.

https://doi.org/10.24215/PCe023 


\section{Introducción}

El creciente impacto en la economía mundial de enfermedades y plagas transfronterizas exóticas en animales y cultivos, muchas de ellas emergentes o reemergentes, han colocado a todas las naciones ante el reto de fortalecer sus capacidades defensivas para disminuir la vulnerabilidad ante desastres sanitarios de grave impacto económico, social y político. Esto ha sido reconocido por organismos internacionales, como la Organización Mundial de la Sanidad Animal (OIE), la Organización Mundial de la Salud (OMS) y la Organización de las Naciones Unidas para la Agricultura y la Alimentación (FAO).

Las consecuencias de distintos brotes de enfermedades en los animales han ocasionado pérdidas cercanas a un tercio de las exportaciones mundiales de carne, equivalente a 6 millones de TM (10.000 millones de USD), excluyendo los costos de las medidas de control y su impacto en los productores, consumidores y la industria animal (FAO, 2005). En el caso de las plagas agrícolas, basta señalar que la presencia en el Caribe de Maconellicoccus hirsutus Green (Cochinilla Rosada del Hibisco), aún sin afectar a Cuba, ha causado pérdidas ascendentes a más de 138 MM USD (Percedo et al., 2008).

Más del $75 \%$ de las enfermedades emergentes identificadas en los últimos años son zoonosis (King, 2004), lo que reafirma la necesidad de fortalecer los preparativos para enfrentar estas amenazas (OMS, 1994 y 1996). El cambio climático global no solo ocasiona el incremento de las tierras áridas y desérticas, sino que también aumenta las enfermedades de los animales y las plagas agrícolas, con los consiguientes perjuicios a las economías de los países (FAO, 2005).

La FAO, la OMS y la OIE han mancomunado esfuerzos y hecho un Ilamamiento a la comunidad internacional para reducir la vulnerabilidad ante las enfermedades transfronterizas, mediante el empleo de estrategias específicas. Estas organizaciones coinciden en señalar que su control efectivo debe estar incluido, como un Bien Público Internacional, entre las Metas de Desarrollo del Milenio (Lubroth, 2005). Más recientemente, en septiembre de 2015, la Asamblea General de la ONU acordó, por consenso de todos los líderes del mundo, el documento: "Transformando nuestro mundo: la Agenda 2030 para el Desarrollo Sostenible". Dicha agenda comprende los Objetivos de Desarrollo Sostenible (ODS) posterior a 2015, con un plan de acción a favor de las personas, la protección del planeta, la seguridad alimentaria y la prosperidad para todos; además tiene la intención de fortalecer la paz universal y el acceso a la justicia para erradicar la pobreza (ONU, 2015).

En Cuba, en el 2005, se pone en vigor la Directiva No. 1 del Consejo de Defensa Nacional, donde se establece que las epidemias, epizootias y epifitias son peligros que pueden crear situaciones de desastres que afecten la seguridad nacional. Además, se destaca que la preparación de los recursos humanos y el aseguramiento de la completa disposición técnica para garantizar la alerta temprana, el diagnóstico precoz y la respuesta rápida es parte de la etapa de preparativos incluida en el Ciclo de Reducción de los Desastres (CDN, 2005).

Como resultado de la organización y planificación de las acciones previstas por nuestro Sistema de Medidas de Defensa Civil (EMNDC, 2006), todos los sectores de la sociedad cubana disponen de una vasta experiencia en el enfrentamiento y respuesta a desastres de todo tipo, en particular a desastres sanitarios, dada la frecuencia con que se ha sufrido su impacto: la Enfermedad de Newcastle en 1961-1962 y 1969, la Peste Porcina Africana (PPA) en 1971 y 1980, la Enfermedad Hemorrágica Viral del Conejo (EHVC) en 1993 y 1997, la reemergencia de la Peste Porcina Clásica desde 1993, la introducción de la Gastroenteritis Transmisible 
Porcina y Disentería Invernal Bovina en 2003 y las plagas agrícolas de la Roya de la Caña de Azúcar, el Moho azul del Tabaco, el Vaneado del Arroz, Thrips palmi y la Broca del Café (Percedo et al., 2007).

Sin embargo, en este entorno, es preciso garantizar la continuidad de la preparación y la capacidad de respuesta ante desastres sanitarios, así como el fortalecimiento de la cooperación intersectorial y multidisciplinaria. Por ello, en el año 2006 se creó el Centro de Capacitación para la Reducción de Desastres Sanitarios en Animales y Plantas (CEDESAP), adscrito al Centro Nacional de Sanidad Agropecuaria (CENSA) y auspiciado por el Ministerio de Educación Superior (MES) y el Estado Mayor Nacional de la Defensa Civil (EMNDC) de Cuba. Su misión está dirigida a la capacitación, la investigación y la transferencia tecnológica con vistas al fortalecimiento de las capacidades defensivas sanitarias frente a las enfermedades exóticas y cuarentenadas, en el ámbito agropecuario, piscícola y forestal (Percedo et al., 2008).

El CEDESAP tiene como antecesor el Centro Veterinario para la Prevención en Casos de Desastres (CVPCD), fundado en 1991 en el marco del Decenio Internacional para la Reducción de Desastres Naturales (DIRDN), convocado por la Organización de las Naciones Unidas (ONU) y cuenta con un centro virtual que da soporte a su trabajo: REDesastres, primera red telemática del sector agropecuario cubano, conformada todas las Direcciones y Laboratorios Provinciales de Diagnóstico del Instituto de Medicina y de Sanidad Vegetal, centros de investigación y universidades agropecuarias del país donde se forman los futuros médicos veterinarios y agrónomos, así como instituciones de los Ministerios de Educación Superior (MES), Agricultura (MINAG), Salud Pública (MINSAP), Pesca (MIP) y Ciencia, Tecnología y Medio Ambiente (CITMA) y Defensa Civil.

La decisión de reestructurar al antiguo CVPCD para dar paso al CEDESAP-REDesastres, respondió a los siguientes factores: (Percedo et al., 2007)

- El incremento del riesgo sanitario por enfermedades y plagas exóticas a escala mundial y la necesidad de mitigar los desastres sanitarios.

- La urgencia de que las universidades y la sociedad en su conjunto trabajaran sostenidamente en la preparación de recursos humanos para enfrentar los desastres.

- El reconocimiento nacional al Centro Nacional de Sanidad Agropecuaria (CENSA) por su desempeño como centro de referencia nacional para el diagnóstico de enfermedades exóticas y capacitación técnica, avalado en 2002 por la FAO al designarlo centro colaborador para la preparación ante emergencias por enfermedades transfronterizas de animales en la región del Caribe.

- La posibilidad de extender al sector agrícola las experiencias adquiridas en manejo de desastres en el ámbito de la medicina veterinaria.

- El potencial de las Tecnologías de la Información y las Comunicaciones (TIC), así como de la gestión de información, para fomentar el trabajo colaborativo en red y la actualización y socialización de conocimientos.

REDesastres cumple así el propósito de brindar soporte informacional a todos los miembros de la red como factor clave para la actualización, el conocimiento y la toma de decisiones acertadas. 
Hoy día la información y el conocimiento se han convertido en las principales fuerzas productivas de las organizaciones y los países, por ello, divulgar las experiencias del trabajo de gestión de información en REDesastres constituye el objetivo del presente artículo.

\section{Desarrollo}

Con vistas al fortalecimiento de las actividades dirigidas a la capacitación y preparación de todos los actores y los sectores del país involucrados en la reducción de desastres sanitarios en los sectores agropecuario, piscícola y forestal, y a su vez, lograr su participación activa, se constituyó REDesastres, primera red virtual de amplia cobertura nacional que se apoya en las herramientas de la gestión de información y los recursos de las tecnologías de la informática y las comunicaciones (TIC'S).

REDesastres unifica los esfuerzos de sectores afines a sus objetivos para potenciar la cooperación y la actualización permanente de técnicos, productores y decisores de la esfera agropecuaria cubana.

Las ideas preliminares del proyecto se promovieron en el Centro Nacional de Sanidad Agropecuaria (CENSA) y desde sus inicios participaron profesionales en salud animal y ramas afines, junto con otros funcionarios de la Dirección de Protección de Plantas del centro.

En sus inicios, para impulsar la creación de REDesastres, se establecieron alianzas con la Facultad de Medicina Veterinaria de la Universidad Agraria de la Habana (UNAH) y el resto de las antiguas filiales del CVPCD, y se convocaron sendos talleres en el CENSA y la Universidad de Granma (UDG) en el año 2005. Concurrieron a estos talleres representantes de los servicios oficiales de sanidad animal y vegetal del país y de todas las instituciones docentes y de investigación, así como del MES y la Defensa Civil.

Además de servir de marco para divulgar experiencias de trabajo relativas a la respuesta a desastres en el ámbito agropecuario, ambos talleres sirvieron para exponer los objetivos, la estrategia y el plan de acción del nuevo proyecto, y para forjar las alianzas necesarias para dar inicio a REDesastres.

En la actualidad participan en la red especialistas en epidemiología, microbiología, manejo de plagas, calidad, información científico-técnica, matemática e informática del CENSA. Inicialmente contaba con una comunidad virtual de 95 usuarios, pero ahora la red llega a más de 510 destinos, 432 nacionales distribuidos en todas las provincias y 35 especialistas de siete países del área latinoamericana. Entre ellos hay investigadores (72), profesores (118), especialistas de la producción (68), funcionarios (66) y directivos (70) de centros de investigación y universidades agropecuarias, instituciones productivas y de servicios técnicos, las direcciones técnico-administrativas y los laboratorios de diagnóstico de los servicios oficiales de sanidad animal y vegetal, la Defensa Civil, así como los sectores productivos pertenecientes a los Ministerios de Educación Superior (MES), Agricultura (MINAG), Salud Pública (MINSAP), Pesca (MIP) y Ciencia, Tecnología y Medio Ambiente (CITMA), entre otros.

Se destaca la participación en REDesastres de los futuros médicos veterinarios y agrónomos las universidades del país, entre ellas, la Universidad Agraria de la Habana (UNAH), la Central de Las Villas (UCLV), de Sancti Spíritus (CUSS), de Ciego de Ávila (UNICA) y de Granma (UDG). 
Desde el punto de vista informático, REDesastres se basa en un sistema computarizado en Microsoft Access 2007 o superior, con una base de datos donde se registran los datos primarios referidos al análisis de la probabilidad de ocurrencia de las enfermedades consideradas desastres sanitarios, la identificación y la caracterización de los lugares de importancia para la introducción/diseminación de enfermedades (objetivos con peligro biológico), así como la evaluación de los diferentes tipos de vulnerabilidad (de la población animal, social, ecológica, económica) y otros factores considerados como premisas de desastres sanitarios.

El funcionamiento de REDesastres se apoya en una lista de distribución electrónica (redesastres-L@censa.edu.cu), la intranet del centro (http://intranet.censa.edu.cu/cedesap) y el sitio web del CEDESAP (http://www.censa.edu.cu/cedesap). Se incluye, además, como un destino la lista de la red Seguridad alimentaria (seguralim@ica.co.cu) y a través de la página Web de la Sociedad Cubana de Medicina Veterinaria de Desastres (http://www.scmvd.sld.cu); también se puede acceder al sitio del CEDESAP-REDesastres.

El sitio del CEDESAP permite acceder a toda la información nacional e internacional recopilada y distribuida sobre el tema, así como a los mensajes circulados desde el inicio a través de la lista de correo electrónico, incluyendo documentos adjuntos (*.doc, *.pdf, *.htm, *.mht, etc.). Los mensajes recientes aparecen en la página de inicio en la sección de Noticias.

REDesastres emplea una filosofía de trabajo basada en el intercambio intensivo de información y conocimientos, lo que ha permitido a sus miembros reducir la incertidumbre, reaccionar mejor ante los cambios del entorno y elevar la cultura sobre la gestión de riesgos y la reducción de desastres.

El empleo de las herramientas de gestión de información en REDesastres ha propiciado la participación, capacitación y educación activas de todos aquellos insertados en la prevención y mitigación de desastres sanitarios. La divulgación de las últimas informaciones y noticias relevantes en este campo ha posibilitado una adecuada transferencia a los que deben tomar decisiones informadas y ha generado nuevos conocimientos y propuestas para el desarrollo de la humanidad.

\section{La gestión de información en REDesastres}

El avance y la generalización del conocimiento dependen, en gran medida, de las posibilidades de acceso a la información y la capacidad para procesarla y difundirla. El gran reto es cómo convertir en conocimiento útil la avalancha de información que se propaga a través de los diferentes medios de comunicación y, también, cómo aprovechar el proceso de generación y apropiación del conocimiento para inducir procesos dinámicos de aprendizaje social, a través de los cuáles el conocimiento crea o fortalece capacidades y habilidades en las personas $u$ organizaciones que se lo apropian, convirtiéndose en factor de cambio en la sociedad, en sus instituciones, o en las empresas (Romero, 2002). Es aquí donde la gestión de información asume el reto de controlar toda la información que se produce y compete a los diversos roles y actores sociales, agregándole valor para optimizar la toma de decisiones.

Los nuevos paradigmas del trabajo colaborativo en red están teniendo un profundo impacto en todos los sectores de la actividad humana y han modificado significativamente la sistematización y distribución de la información, prácticamente en el mismo instante en que ésta se genera, haciendo posible su socialización entre la comunidad. 
En REDesastres la gestión de información ha posibilitado el análisis y el procesamiento de diversos recursos de información relevantes del entorno. Específicamente su misión es la de capturar, evaluar, procesar, diseminar, añadir valor y darle seguimiento a la información científica tecnológica procedente de los ámbitos externo e interno con absoluta inmediatez, creando así un flujo interactivo de información y conocimiento que llega a todos los miembros de la Red, en aras de propiciar el cumplimiento de su misión primordial: la Reducción de Desastres Sanitarios en Animales y Plantas. Las fuentes informativas que se emplean son: organismos sanitarios y agencias noticiosas especializadas internacionales y nacionales, publicaciones científicas, reportes y alertas informativas, todas ellas relativas a la prevención, el diagnóstico y el control de enfermedades emergentes, reemergentes y transfronterizas, y la gestión de desastres.

El equipo de trabajo que se encarga de esta labor está integrado por dos especialistas, maestros en ciencia y en gestión de información. De esta forma, el manejo, la conservación y la diseminación de la información está a cargo de profesionales que han desarrollado capacidades y destrezas, aplicando diversas técnicas gerenciales y agregando valor a la información.

Actualmente han circulado más de 1.376 mensajes a todos los miembros de la red; estas noticias o informaciones, en muchos casos, se acompañan de comentarios técnicos que posibilitan ampliar y debatir los conocimientos para la mejor toma de decisiones en el desempeño del trabajo técnico y profesional.

\section{Aportes de la Gestión de Informacion en REDesastres}

La permanente vigilancia de la información que se emite sobre enfermedades emergentes, reemergentes y transfronterizas de animales y cultivos, así como la diseminación inmediata de las notificaciones sanitarias de la OIE y de medios no oficiales, son aspectos de relevante prioridad en el trabajo. También noticias sobre la situación sanitaria internacional, los programas de prevención y control, el estado del arte en diagnóstico, las zoonosis y enfermedades reemergentes, candidatos vacunales y nuevas vacunas, propiedades de cepas circulantes, así como temas relacionados con las características de las enfermedades, sus medidas de prevención, la bioseguridad y comentarios de expertos nacionales e internacionales sobre su impacto económico y social, entre muchas otras.

Las informaciones técnicas, puestas a disposición de los decisores, incluyen las comunicaciones a la Organización Mundial del Comercio hechas por Cuba acerca de los requisitos sanitarios a importaciones con riesgo, además de normativas y recomendaciones.

A través de REDesastres se han desarrollado importantes intercambios de información y experiencias dando lugar a foros de discusión sobre Influenza aviar (IA), Peste Porcina Clásica, Ralstonia solanacearum, Ébola, Cambio climático, Programas de prevención y control de enfermedades adoptados en diferentes países, que han servido para fortalecer su prevención a través de la capacitación y actualización de estas importantes enfermedades y plagas. Actualmente se realizan otros foros y comunicaciones sobre la presencia de IA en nuevos países y la notificación de casos de infección o muerte en humanos, el Dengue, el Zika y el Chikungunya, la Resistencia a los antibióticos, temáticas a las que se le da permanente vigilancia. 
Es de destacar que la página web de la red cuenta con una biblioteca virtual (InfoDesastres), con más de 400 documentos recopilados a texto completo, de acceso a todos los usuarios nacionales y de mucha utilidad para la consulta especializada.

\section{Ventajas del trabajo de Gestión de información}

En REDesastres el trabajo de gestión de información ofrece evidentes ventajas en aspectos claves, tales como:

- Agiliza la comunicación, el intercambio y la socialización de informaciones de interés, una necesidad en la gestión de desastres y en los preparativos para la respuesta: alerta temprana, diagnóstico precoz y respuesta rápida.

- Facilita la preparación de los recursos humanos e incrementa las posibilidades de intercambio de conocimientos y experiencias.

- Contribuye a una adecuada percepción de los riesgos y al desarrollo de una cultura de la protección (seguridad) frente a los diferentes peligros de desastres.

- Proporciona una vía para la diseminación rápida y uniforme de información sanitaria actualizada, que contribuye a potenciar el esfuerzo mancomunado de todos sus miembros; además, representa un aporte valioso a la cooperación de los diversos actores sociales que intervienen en la reducción de desastres sanitarios en animales y plantas, sin descontar las zoonosis.

El desempeño de REDesastres ha recibido reconocimiento internacional al ser seleccionada en el Concurso de "Casos exitosos en el uso de tecnologías de información y comunicación para la investigación e innovación tecnológica agropecuaria en América Latina y el Caribe", convocado por INFOTEC (Centro de Investigación e Innovación en Tecnologías de la Información y Comunicación), el Sistema de Información Científica y Tecnológica del Sector Agropecuario en las Américas y el Instituto Interamericano de Cooperación de las Américas (IICA, FORAGRO, 2007). También recibió en el año 2016 el Premio del Ministerio de Educación Superior de Cuba al "Resultado ya aplicado de mayor aporte a la defensa del país" y ha sido presentado en numerosos congresos y foros nacionales e internacionales.

Algunos ejemplos de informaciones circuladas en REDesastres se exponen a continuación, como muestra del amplio espectro y de la importancia de los temas que se abordan. El número de impacto significa las veces que ha sido consultada la noticia o el artículo a través del sitio CEDESAP-REDesastres:

- Impactos: 87 [redesastres-I] Shanghái registra su primer caso de gripe aviar de tipo H7N9 en un ser humano Impactos: 89 [redesastres-I] El cambio climático, la mayor amenaza del siglo XXI

- Impactos: 79 [redesastres-I] Zoonosis emergentes alimentarias

- Impactos: 87 [redesastres-I] Vacunas efectivas contra la PPA??

- Impactos: 63 [redesastres-I] RV: ROU 02-12-15 OIE Alerta - Enfermedad de Newcastle 
- Impactos: 62 [redesastres-I] RV: FRA 02-12-15 OIE Alerta - Influenza aviar altamente patógena

- Impactos: 63 [redesastres-I] Continua difusión del virus Zika

- Impactos: 81 [redesastres-l] Laringotraqueítis infecciosa: un problema que no desaparece

- Impactos: 52 [redesastres-I] RV: MAR 02-11-15 OIE Alerta - Fiebre aftosa

- Impactos: 77 [redesastres-I] OIE. Establecer sistemas de sanidad animal resilientes para reducir las amenazas biológicas

- Impactos: 56 [redesastres-I] Zoonosis: Hepatitis E

- Impactos: 63 [redesastres-I] EEUU: Posible vía introducción DEP

- Impactos: 118 [redesastres-I] Nota de prensa de la OIE: Hacia una mejor vigilancia del uso de antibióticos en el ámbito de la sanidad animal

- Impactos: 111 [redesastres-I] Bacterial leaf blight, rice - Bangladesh: (DA)

- Impactos: 130 [redesastres-I] Invasive mosquito - USA (09): (CA)

- Impactos: 119 [redesastres-I] Rabia, problema sanitario en Haití

- Impactos: 62 [redesastres-I] Agricultura y cambio climático: el reto de alcanzar la seguridad alimentaria

- Impactos: 66 [redesastres-I] Nueva vacuna contra gripe aviar

- Impactos: 89 [redesastres-I] Comisión Europea: Guía sobre el uso prudente de los antimicrobianos en veterinaria

- Impactos: 80 [redesastres-I] La avicultura latinoamericana define la estrategia contra la influenza aviar

- Impactos: 75 [redesastres-I] Mancha naranja del arroz, Costa Rica

- Impactos: 82 [redesastres-I] Hallan en focas un virus parecido al de la hepatitis $A$

- Impactos: 97 [redesastres-I] Leptospira vaccines

- Impactos: 97 [redesastres-I] La FAO alerta de la aparición de la gripe aviar en África occidental/Más problemas para África

- Impactos: 75 [redesastres-1] Cambio climático: el mes pasado fue el junio más cálido desde 1880

- Impactos: 66 [redesastres-I] Alemania 27-07-15 OIE - Alerta - Influenza aviar altamente patógena

- Impactos: 87 [redesastres-I] RV: PRO/AH/EDR> Brucellosis - USA: (NY) imported, laboratory exposures

- Impactos: 83 [redesastres-I] EEUU está desarrollando vacuna $100 \%$ efectiva contra la gripe aviar en pollos

- Impactos: 100 [redesastres-I] Ebola/ epidemiología/ investigaciones 
- Impactos: 100 [redesastres-I] Influenza aviar: China, H7N9

- Impactos: 78 [redesastres-I] EEUU: Actualización del Coronavirus Entérico Porcino

- Impactos: 69 [redesastres-I] Desastres naturales, más letales en el primer semestre de 2015

- Impactos: 135 [redesastres-I] PRO/PL> Citrus bark cracking viroid, hop - Slovenia: new host

- Impactos: 82 [redesastres-I] Vulnerable América Latina ante el cambio climático

- Impactos: 77 [redesastres-I] En París, dos mil científicos discuten soluciones frente al cambio climático

\section{Consideraciones finales}

En el contexto actual, los crecientes riesgos a escala global de emergencia o reemergencia de enfermedades en animales y cultivos por la introducción de enfermedades y plagas exóticas y cuarentenadas en animales y cultivos, incluyendo las zoonosis, constituyen continuas amenazas de desastres sanitarios de grave impacto económico, social, político y para la salud pública, además de poseer un alarmante potencial transfronterizo a amplias regiones geográficas.

Fortalecer la cooperación multisectorial e interdisciplinaria para enfrentar exitosamente estos desastres constituye un pilar fundamental de la estrategia nacional de Cuba que demanda optimizar la actuación de los diferentes actores sociales involucrados y en donde la vigilancia informativa deviene en un factor permanente, necesario e ineludible.

Dada la compleja situación sanitaria internacional, la actuación de REDesastres ha estado encaminada a dar respuesta a las indicaciones de los Ministros de Salud Pública y de la Agricultura de Cuba dirigidas a evitar o mitigar los riesgos de afectación a la seguridad nacional en sus diferentes etapas: Prevención, Preparativos, Respuesta y Recuperación.

El empleo de las herramientas de la gestión de información en REDesastres ha contribuido con el cumplimiento de su misión y se ha convertido en un imperativo para la estrategia de reducción de desastres sanitarios en Cuba con enfoque a "UNA SALUD".

\section{Referencias bibliográficas}

IICA, FORAGRO. (2007). Casos exitosos en el uso de tecnologías de información y comunicación para la investigación e innovación agropecuaria en América Latina y el Caribe. San José: IICA, FORAGRO. Recuperado de http://infoagro.net/infotec/exitosos/

CDN (Consejo de Defensa Nacional). (2005). Directiva No. 1 del Vicepresidente del Consejo de Defensa Nacional para la Planificación, Organización y Preparación del País para Situaciones de Desastres. 1ro de junio, La Habana, Cuba.

EMNDC (Estado Mayor Nacional de la Defensa Civil). (2006). 45 Aniversario Defensa Civil de Cuba. Ponencia presentada en VII Cong. Int. sobre Desastres, Palacio de Convenciones, La Habana, Cuba. 
King, L. J. (2004). Zoonosis y patógenos emergentes de importancia para la salud pública. Rev. sci. tech. Off. int. Epiz., 23 (2) 429-433.

Lubroth, J. (2005). Programa Global para el Control Progresivo de las Enfermedades Transfronterizas de los Animales (GF-TAD's). Ponencia presentada en Reunión de la Región de Mesoámerica y del Caribe. VII Congreso Centroamericano y del Caribe de Porcicultura.

ONU (Organización de las Naciones Unidas). (2015). La Asamblea General adopta la Agenda 2030 para el Desarrollo Sostenible. Recuperado de http://www.un.org/sustainabledevelopment/es/2015/09/la-asamblea-general-adopta-laagenda-2030-para-el-desarrollo-sostenible/

FAO (Organización de las Naciones Unidas para la Agricultura y la Alimentación). (2005). Disminuyen las exportaciones mundiales de carne a causa de los brotes de enfermedades animales. Recuperado de http://www.fao.org/ newsroom/es/news/ 2004/37967/index.html

OMS (Organización Mundial de la Salud). (1994). Emerging and reemerging infections diseases: WHO responds to a global threat. Comunicado de Prensa WHO/37, 26 de abril. Bol. Ofic. Sanit. Panam. 116 (6) 545.

OMS (Organización Mundial de la Salud). (1996). Creación de una nueva unidad en la OMS. Foro Mundial Salud 17 (1) 99.

Percedo, M.I., Rodríguez, M.G., Alfonso, P., Abeledo, M.A., Canales, H., González, I., Navarro, L. (2008). CEDESAP y REDesastres. Una contribución a la preparación y gestión intersectorial y multidisciplinaria para la reducción de desastres sanitarios en animales y plantas REDVET. Revista electrónica de Veterinaria 9 (11). Recuperado de http://www.veterinaria.org/revistas/redvet/n111108.html

Percedo M.I, Rodríguez M.G., Alfonso P., Frías, M.T., González I., Tablada L.M. y Encinosa A. (2007). REDesastres, una contribución cubana a la gestión de desastres sanitarios en animales y plantas. Recuperado de http://cedesap.censa.edu.cu/index.php/publicaciones/category/6cedesap-redesastres

Romero, A. (2002). Las redes de información y su importancia para la investigación científica. Revista Venezolana de Gerencia 7 (julio-noviembre). Recuperado de http://www.redalyc.org/articulo.oa?id=29001906> ISSN 1315-9984 